\title{
Mechanical Analysis of Mining Stress Transfer on Isolated Island Face in Extra-Thick Fully Mechanized Top-Coal Caving Mining
}

\author{
Min Tu, ${ }^{1}$ Qingwei Bu $\mathbb{D}^{1,2}$ Baojie Fu, ${ }^{1}$ and Yu Wang ${ }^{1}$ \\ ${ }^{1}$ Key Laboratory of Safety and High-Efficiency Coal Mining of Ministry of Education, Anhui University of Science and Technology, \\ Huainan 232001, China \\ ${ }^{2}$ School of Mining and Coal, Inner Mongolia University of Science and Technology, Baotou 014010, China
}

Correspondence should be addressed to Qingwei Bu; buqw1988@imust.edu.cn

Received 4 June 2020; Revised 28 July 2020; Accepted 21 August 2020; Published 8 September 2020

Academic Editor: Zhijie Wen

Copyright ( $92020 \mathrm{Min}$ Tu et al. This is an open access article distributed under the Creative Commons Attribution License, which permits unrestricted use, distribution, and reproduction in any medium, provided the original work is properly cited.

\begin{abstract}
The mining spatial structure of isolated island face in extra-thick fully mechanized top-coal caving mining is unique, which leads to a complex mining stress distribution and serious safety hazards. In this study, combined with a specific engineering example, the mining stress distribution characteristics of isolated island face are expounded, and a bearing structural mechanical model of the continuous beam of overlying strata is established using elastic-plastic mechanics theory. The mechanical equations of the mining stress distribution and failure depth of coal-rock mass are then obtained. Comparison of theoretical calculation results with numerical simulation and field measurement results shows basically consistent stress distribution characteristics. The derived mechanical equations can provide an estimation method for the analysis of mining dynamics on isolated island face in extra-thick fully mechanized top-coal caving mining. The following conclusions are acquired. The coal-rock mass should bear not only the lateral mining superposition influence but also the advance mining influence in front of the coal wall, so the isolated island face is in the complex environment of multiple mining stress superposition. In the mining process, the maximum advance mining stress concentration factor is 4.0-6.0 and is located at the upper and lower ends of the isolated island face. The lateral mining failure depth of the coal wall of the isolated island face increases by $2.0-5.0 \mathrm{~m}$ under the influence of advance mining. Therefore, compared with the nonisolated island face, the mining pressure appearance is intense. The mining influence in the range of $20-30 \mathrm{~m}$ of the upper and lower ends is intense, and the mining stress in this area is characterized by "cone distribution." This zone is an important hidden danger area with coal-rock mass mining instability on isolated island face, which requires special attention to avoid mining disasters. According to the analysis of the influencing mining factors and laws of isolated island face, it is concluded that the longer the isolated island face size is, the closer the goaf size on both sides of the isolated island face is, the smaller the coal seam buried depth is, the better the mechanical conditions of coal and rock medium are, and the smaller the mining height of coal seam is, the more favorable the safe mining of isolated island face is.
\end{abstract}

\section{Introduction}

The safe mining of isolated island face is of great significance to the safety production and economic benefit of coal mines. In the process of isolated island face formation, the overlying strata are affected by the lateral mining superposition influence of bilateral goafs. Consequently, the bearing pressure of coal-rock mass is more serious than that of nonisland working face, and the pressure step distance of isolated island face is smaller than that of nonisolated island face. Although failure occurs in coal-rock mass at the lateral edge of isolated island face due to mining, the coal-rock mass of the isolated island face and the overlying strata of the fracture zone in goafs still maintain continuous and complete structural characteristics and have good continuous bearing conditions and mining force transmission [1-9].

The bearing pressure of an isolated island face comes from its own and the rock load carried by the overlying strata slabs or beams on both sides. Therefore, the mining influence of isolated island face is more complex and severe than that of nonisolated island face. The safe mining of islands under the influence of strong mining has been concerned for a long 


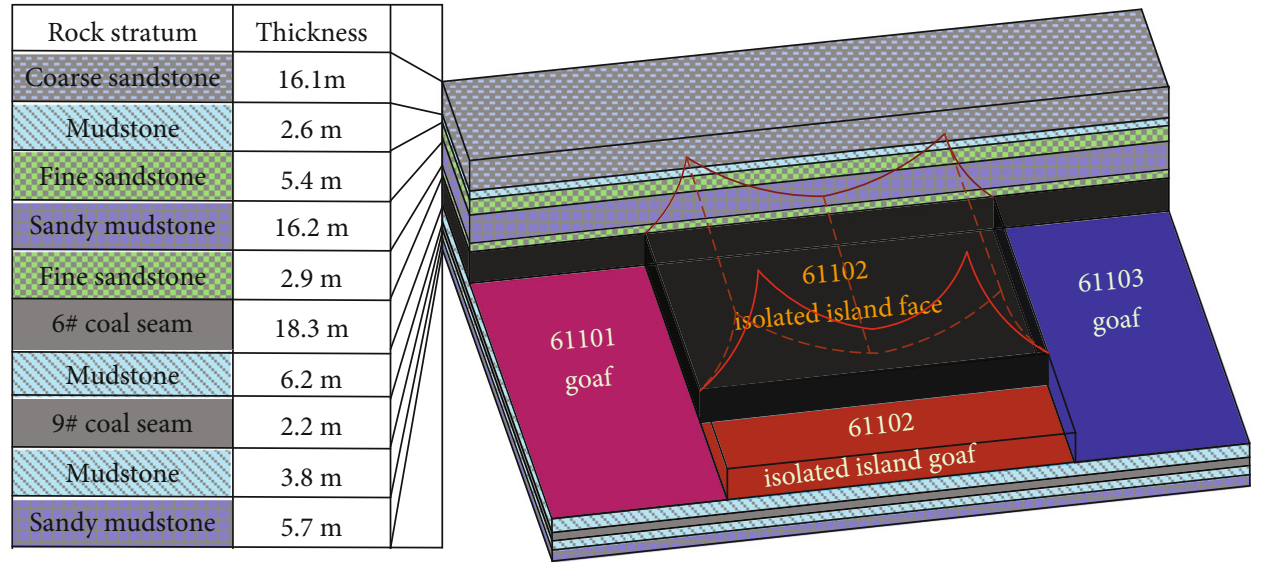

FIGURE 1: Space arrangement and rock occurrence conditions of the isolated coal face in fully mechanized caving mining.

time, and the research has made great progress. For instance, Jiang et al., Shi et al., Cheng et al., and Hou et al. classified the overlying stratum space structure of isolated island face stopes into "O," "S," and "C" types [1-5]. They also studied the mining stress transfer and periodic weighting rules of the surrounding rock of isolated island face and provided guidance for the control of overlying stratum weighting. Dou et al. and Cao and others regarded the spatial structure of isolated island overlying strata as "F" and "T"-type bearing structure; they analyzed the spatial force transfer and induced rock burst mechanism of the isolated island overlying strata, which is of guiding significance for the mining pressure control of isolated island face [6-8]. Wang et al., Jiang et al., Li et al., and Liang et al. studied the stratum pressure behavior characteristics of isolated island face with overburden, hidden danger, and coal pillar mining instability [912]. Zhao and Kang and Xu et al. compared and analyzed the differences between top-coal caving isolated island face and top-coal caving face and discussed the movement characteristics of top coal and roof in the mining process of a top-coal caving isolated island face $[13,14]$. Wang et al., Jia et al., and Chang et al. used the hyperbolic mechanical model of stress load transfer in continuous media to analyze the mechanical failure of coal-rock mass at the edge of goafs and guide the engineering practice [15-18]. Zhang and Tu analyzed the mining stress distribution and failure characteristics of an isolated island face affected by multiple mining in view of the engineering conditions of the isolated island face formed by the goaf of the upper and lower coal seams. They aimed to provide an engineering technology reference for the safe mining of isolated island face under similar special engineering conditions [19]. Zhou and Li Hong used numerical simulation and on-site engineering practice to analyze the problem of roof caving on an isolated island face of large mining height [20]. Wang et al. analyzed the distribution law of the bending moment of isolated island face roof on the basis of a thin-plate mechanical model, and the mining stress concentration and failure evolution of the surrounding rock were studied through numerical simulation [21].

However, a mechanical analysis of complex mining effects, such as mining failure depth, the complex characteristics of mining stress distribution, and the intensity of the mining stress concentration of the coal-rock mass in isolated island face in fully mechanized top-coal caving mining, should be conducted. The safety production of isolated island face in fully mechanized top-coal caving mining urgently needs relevant theoretical research as technical guidance. The current study bases on the analysis of the bearing capacity characteristics of coal-rock mass of isolated island face in Tangjiahui Coal Mine and the analysis of the force-bearing characteristics of the coal-rock mass through numerical simulation. A mechanical model analysis of mining bearing in the isolated island face is performed. The mining load-force transfer mechanism of the coal-rock mass is studied. This study provides an estimation method for the calculation of mining stress distribution of the isolated island face in fully mechanized top-coal caving mining and plays a certain role in engineering guidance for the safe production of isolated island face.

\section{Mining Influence Analysis of the Isolated Island Face in Fully Mechanized Top-Coal Caving Mining}

2.1. Project Overview. The 61102 isolated island face in fully mechanized top-coal caving mining in Tangjiahui Coal Mine has the following characteristics: the average thickness of the main mining 6\# coal seam is $18.3 \mathrm{~m}$, the average dip angle of the coal seam is $2^{\circ}$, the buried depth is $520 \mathrm{~m}$, and the plane layout is shown in Figure 1.

The rock mass presents the mechanical characteristics of low strength and poor cementation under the later geological structure because of the late diagenesis and low maturity of the coal measure strata, which are mostly argillaceous and calcareous cementation. Problems, such as in mine pressure control, support bearing stability, and surrounding rock control of the mining roadway, occur in the mining process of the working face. Therefore, the mining stress distribution and failure in the isolated island face should be studied urgently.

2.2. Analysis of the Bearing Capacity Characteristics of CoalRock Mass. In accordance with the research on the behavior of mining pressure in isolated islands $[19,20]$ and field 
investigation, the preliminary analysis on the bearing capacity characteristics of coal-rock mass under the complex mining influence in the isolated island face indicates the following findings:

(1) As the "bearing and transmitting" structure of the gob on both sides and its overlying strata, the island coal face bears "part" load of the overlying strata on both sides, and the bearing load is higher than that in the nonisolated island face

(2) The characteristics of the spatial bearing structure in the isolated island face lead to the lateral mining superposition influence of bilateral goafs, resulting in intensified mining stress peak value, mining failure, and deep bearing stress distribution. The effects of many factors make the bearing state and force failure of coal-rock mass present complex and strong mining pressure in the isolated island face

(3) Under the stress environment affected by the lateral mining superposition influence of bilateral goafs, the isolated island face mining results in a serious mining influence, overlying stratum pressure, fracture and rotation of the overlying stratum block, and a relatively "positive" mechanical response

(4) The mining intensity, overlying stratum failure, pressure appearance characteristics, and surrounding rock stability of mining roadway of the isolated island face differ from those of a nonisolated island face. Hence, the characteristics of bearing capacity of coal-rock mass under the mining influence of the isolated island face should be analyzed

\section{Simulation Analysis of the Mining Influence Characteristics of the Isolated Island Face in Fully Mechanized Top-Coal Caving Mining}

On the basis of field investigation and analysis, numerical simulation analysis is used to reveal the distribution characteristics of lateral mining superposition influence and the advance mining pressure in the isolated island face. The objective is to provide a strong reference for the mechanical model analysis of mining stress transfer in coal-rock mass regarding mining influence.

3.1. Establishment of a Calculation Model for the Isolated Island Face in Fully Mechanized Top-Coal Caving Mining. This study regards the 61102 isolated island face in fully mechanized top-coal caving mining in Tangjiahui Coal Mine as the object. Numerical simulation is performed, and the model is shown in Figure 2. The model size is $1376 \mathrm{~m}(X)$ $\times 504 \mathrm{~m}(Y) \times 622 \mathrm{~m}(Z)$ with 538776 zones, the top boundary is set as vertical stress constraint, and the lateral four sides and the bottom boundary are set as displacement constraints. The original rock stress is $14.04 \mathrm{MPa}$ (with coal seam buried depth of $520 \mathrm{~m}$ ), and the lateral stress coefficient is 1.0 . The width of the 61102 isolated island face is $235 \mathrm{~m}$; the widths

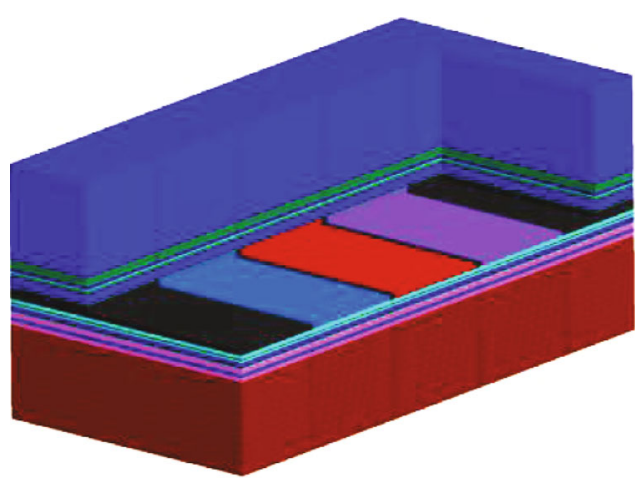

Coal seam

61103 goaf

61102 isolated island face

61101 goaf

Coarse sandstone

Bedrock

Mudstone

Sandy mudstone

Fine sandstone

Medium fine sandstone

FIGURE 2: Numerical calculation model.

of 61103 and 61101 working face are 220 and $240 \mathrm{~m}$, respectively; the width of the lateral boundary medium is $200 \mathrm{~m}$. The specific simulation process is as follows:

(1) The medium is a Mohr-Coulomb model. In accordance with the mechanical test of geological drilling and coring rock in Tangjiahui Coal Mine, the mechanical parameters of coal-rock are obtained through numerical simulation, as shown in Table 1

(2) The boundary stress and displacement conditions are set and the initial stress field balance calculation is completed

(3) The 61101 and 61103 goafs are excavated step by step, and the simulated mining step distance is $18.0 \mathrm{~m}$. On the basis of the double-yield model used in references [22-25], the solution process of goaf filling and compaction is simulated; the mechanical parameters of the goaf media are obtained via simulation inversion calculation $[24,25]$, as shown in Table 2, and the stress-strain relationship of the goaf media is calculated using the stress-strain relationship of fractured rock mass derived by Salamon, as shown in Table 3

(4) After the simulation calculation balance of the 61101 and 61103 goaf compaction, the 61102 isolated island face is excavated step by step. The simulated mining step distance is $12.0 \mathrm{~m}$, and the mining area is at $250 \mathrm{~m}$ in $Y$ direction

(5) After balance calculation, the results of 100-246 $\mathrm{m}$ in $Y$ direction are acquired for mining simulation analysis

3.2. Analysis of Simulation Results. As shown in Figure 3, under the lateral mining superposition influence on both 
TABLE 1: Mechanical parameters of coal-rock.

\begin{tabular}{|c|c|c|c|c|c|}
\hline Medium name & Shear modulus (GPa) & Bulk modulus (GPa) & Cohesion (MPa) & Friction angle $\left({ }^{\circ}\right)$ & Tensile strength $(\mathrm{MPa})$ \\
\hline Medium fine sandstone & 1.62 & 1.71 & 2.6 & 35 & 0.62 \\
\hline Bedrock & 1.04 & 1.14 & 3.3 & 33 & 0.82 \\
\hline Sandy mudstone & 0.91 & 0.86 & 3.3 & 37.5 & 2.25 \\
\hline Coal seam & 0.67 & 0.50 & 1.3 & 28 & 0.54 \\
\hline Fine sandstone & 1.04 & 1.14 & 3.3 & 33 & 0.82 \\
\hline Coarse sandstone & 1.54 & 1.68 & 3.3 & 35 & 0.40 \\
\hline Mudstone & 2.04 & 2.15 & 1.6 & 37.4 & 0.41 \\
\hline
\end{tabular}

TABLE 2: Mechanical parameters of the goaf medium in the doubleyield model.

\begin{tabular}{lcccc}
\hline $\begin{array}{l}\text { Medium } \\
\text { name }\end{array}$ & $\begin{array}{c}\text { Shear } \\
\text { modulus } \\
(\mathrm{GPa})\end{array}$ & $\begin{array}{c}\text { Bulk } \\
\text { modulus } \\
(\mathrm{GPa})\end{array}$ & $\begin{array}{c}\text { Friction } \\
\text { angle }\left(^{\circ}\right)\end{array}$ & $\begin{array}{c}\text { Dilatancy } \\
\text { angle }\left(^{\circ}\right)\end{array}$ \\
\hline $\begin{array}{l}\text { Goaf } \\
\text { medium }\end{array}$ & 7.78 & 5.83 & 12 & 8 \\
\hline
\end{tabular}

TABLE 3: Stress-strain relationship of the goaf medium in the double-yield model.

\begin{tabular}{lccccc}
\hline Strain & Stress $(\mathrm{MPa})$ & Strain & Stress $(\mathrm{MPa})$ & Strain & Stress $(\mathrm{MPa})$ \\
\hline 0.01 & 0.003 & 0.19 & 0.103 & 0.37 & 0.452 \\
0.04 & 0.015 & 0.22 & 0.131 & 0.40 & 0.620 \\
0.07 & 0.028 & 0.25 & 0.166 & 0.43 & 0.910 \\
0.1 & 0.042 & 0.28 & 0.210 & 0.46 & 1.533 \\
0.13 & 0.059 & 0.31 & 0.267 & 0.49 & 3.842 \\
0.16 & 0.079 & 0.34 & 0.343 & 0.50 & 7.122 \\
\hline
\end{tabular}

sides of the isolated island face, the lateral mining failure depth is approximately $11-14 \mathrm{~m}$, the lateral mining stress concentration is $35-39 \mathrm{MPa}$ in the coal-rock mass in the isolated island face, and the bearing stress environment is approximately $20 \mathrm{MPa}$ in the middle of the lateral island coal-rock mass. The stress environment of the lateral coalrock mass edge of the 61102 isolated island face is complex and serious. The simulation results show that the overlying stratum load in the goaf transfers to the solid medium of coal-rock and forms the mining influence. The structure of the large-space stope causes the isolated coal body to be the bearing structure influenced by mining on both sides. With the limited size of the isolated coal body, the lateral mining superposition influence is significant, and the goaf is in an unstable compaction state. Many effects lead to the strong mining pressure appearance in the isolated island face in fully mechanized top-coal caving mining.

As shown in Figure 4, the mining of the 61102 isolated island face aggravates the stress environment of the coalrock mass at the lateral edge of the 61102 isolated island face. The coal-rock mass at the end forms a lateral mining failure depth of approximately $12-18 \mathrm{~m}$ and a lateral mining stress concentration of nearly $62 \mathrm{MPa}$. The middle of the isolated island face forms a load-bearing stress environment of approximately $46 \mathrm{MPa}$. Compared with the 1.5-2.5 advance mining stress concentration coefficient of a nonisolated island face, the advance mining stress concentration coefficient in the mining process of the isolated island face is as high as 3.0-5.0. The mining pressure at the end is most serious. Consequently, the coal body at the end position of the isolated island face becomes the key structure to bear the concentration effect of advance mining stress.

\section{Mechanical Model Analysis of the Mining Bearing Capacity of the Isolated Island Face in Fully Mechanized Top-Coal Caving Mining}

In the analysis of mining dynamic problems, the investigation of the distribution of mining stress and the calculation of mining failure depth require determining the mining stress concentration coefficient (or the peak value of mining stress). However, most determination methods adopt an artificial assumption of mining stress concentration coefficient (the empirical value is 2.0 and 2.5 mostly) or numerical simulation of mining stress peak value. The artificial assumption lacks of a strong basis. Many factors, such as coal seam occurrence conditions, original rock stress environment, and isolated island face layout, affect the mining stress state of isolated island face. This condition leads to the artificial assumption that the mining stress concentration coefficient results in a substantial deviation. Although numerical simulation analysis has evident advantages in revealing the mechanical problems of complex engineering, many problems occur in numerical simulation analysis. Problems include the time-consuming modeling and calculation, the accuracy influence of zone size, the heavy simulation workload of influencing rules and factors, and the high requirements for the numerical simulation operation and analysis of researchers. However, the field measurement lags behind the actual engineering activities due to the constraints of deep mining space conditions, monitoring equipment level, and time conditions. Therefore, mechanical model analysis of the mining influence characteristics of the isolated island face is conducted to realize mechanical theoretical analysis of the mining influence of the isolated island face. This analysis provides a timely and effective calculation method for the mechanical estimation of mining stress concentration and failure depth, which can be used for collaborative analysis with numerical simulation and field measurement. 


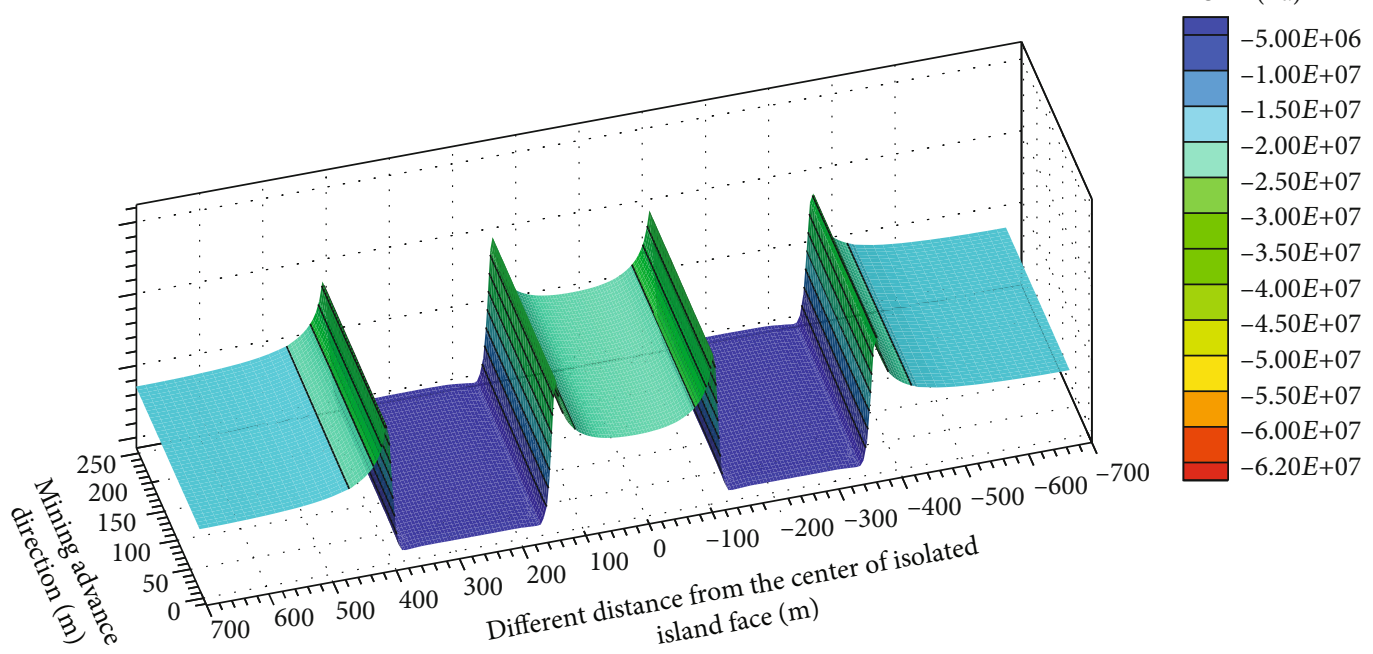

FIGURE 3: Distribution characteristics of lateral mining stress in the isolated island face.

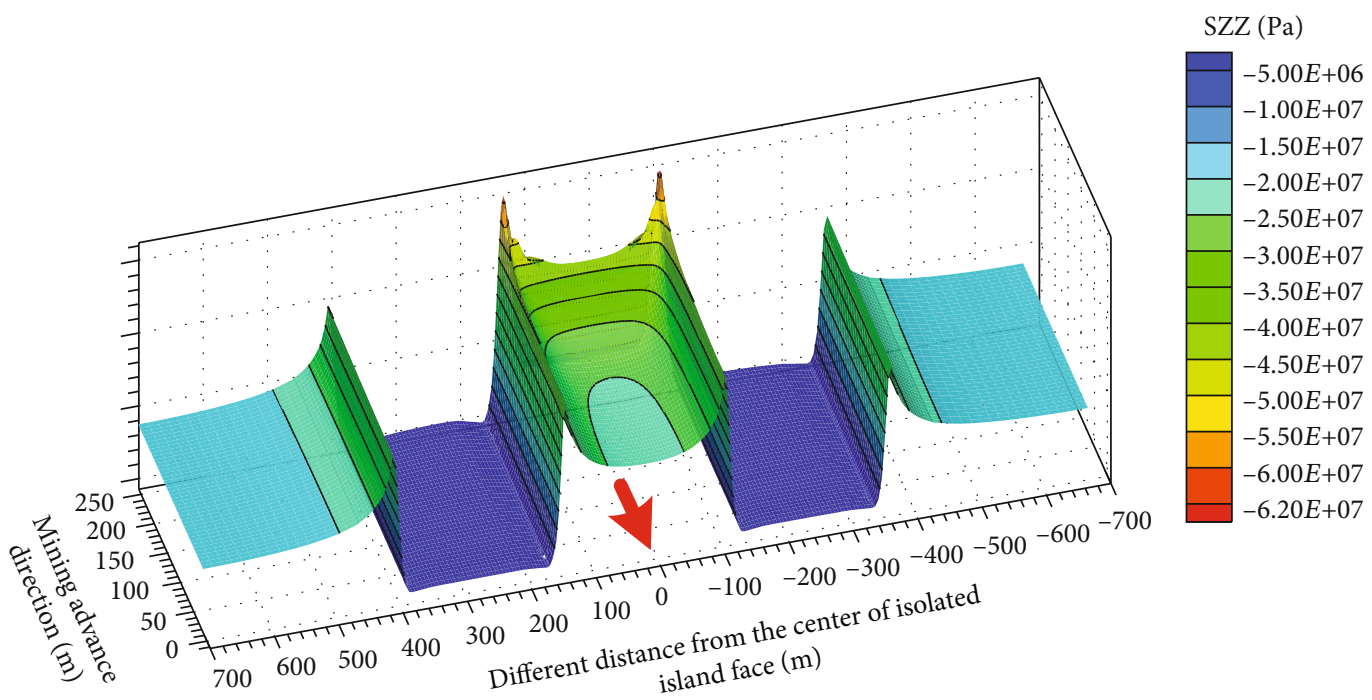

FIGURE 4: Distribution characteristics of advance mining stress in the isolated island face.

4.1. Mechanical Model Construction for the Mining Bearing of the Isolated Island Face in Fully Mechanized Top-Coal Caving Mining. As shown in Figure 5, field investigation and numerical simulation analysis indicate that the coal-rock mass in the isolated island face in fully mechanized caving mining and the coal-rock mass on the outside of the goafs act as the supporting structure of the mining area. They jointly bear the overlying stratum load of the isolated island face and the goafs and maintain the force balance state. In such a mining stress environment, the mining influence of the coal-rock mass in the isolated island face in fully mechanized top-coal caving mining is particularly complex and severe.

On the basis of the theory of mine pressure [1-14, 26, 27], the surrounding rock mass of a goaf is divided into "three horizontal and three vertical zones." The overlying strata of the goaf are gradually broken and developed from the roof boundary to the upward extension depth, forming the caving, fracture, and bending subsidence zones. Among them, the "bending subsidence zone" of synchronous subsidence overlying strata has enhanced continuity and integrity. The overlying strata in this part maintain the continuous bearing effect on the upper stratum load and transfer the mining load of the goaf to the bearing medium of the coal-rock on both sides. Finally, mining stress concentrates in the coal-rock in the isolated island face and the solid coal medium on the outside of the goaf on both sides.

The characteristics of load transfer of the overlying strata in the isolated island face and stress transfer of the surrounding rock in the goaf are similar to those in "the stress structure of slab or beam." From the previous research on the bearing structure of overlying strata in an isolated island [1-9], this paper proposes a mechanical model of the bearing structure of the continuous beam of the overlying strata in an isolated island face in fully mechanized top-coal caving mining, as 


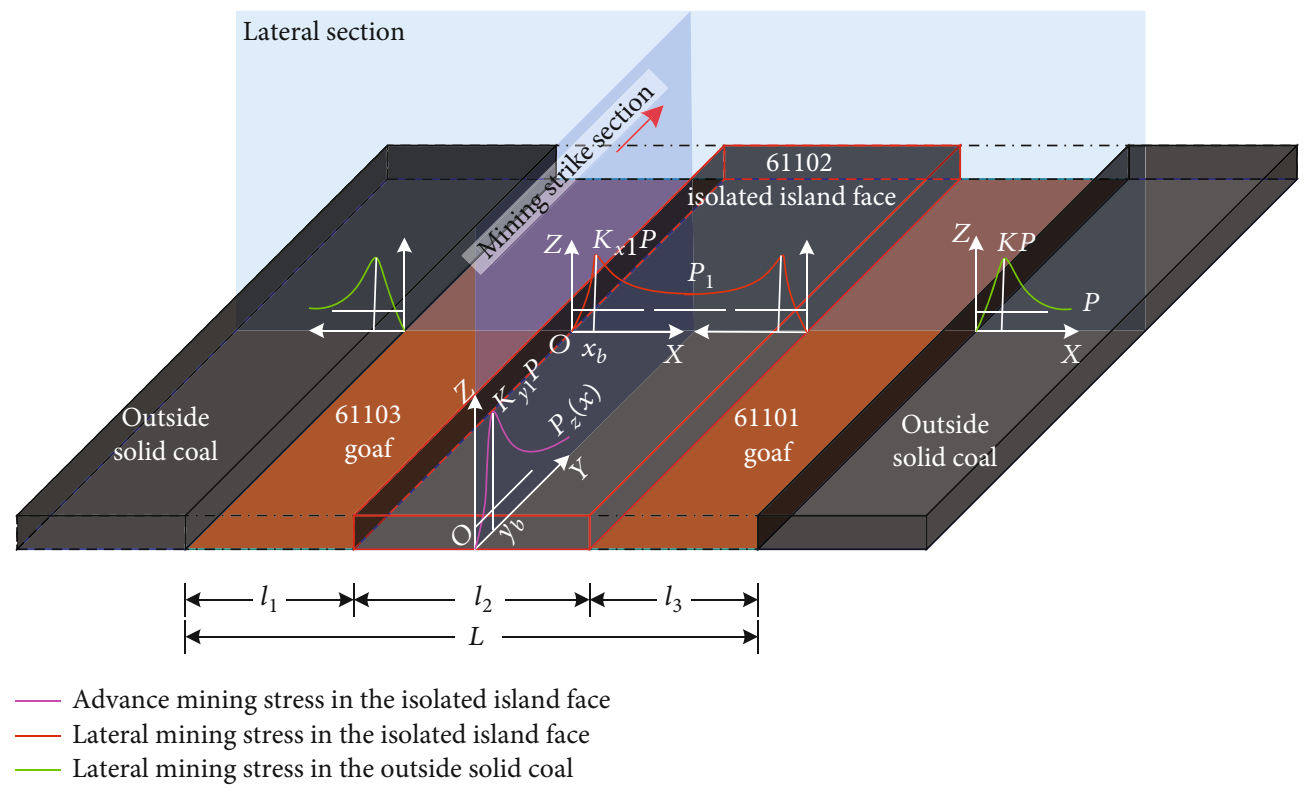

FIGURE 5: Distribution characteristics of mining stress in the coal-rock mass in the isolated island face in fully mechanized top-coal caving mining.

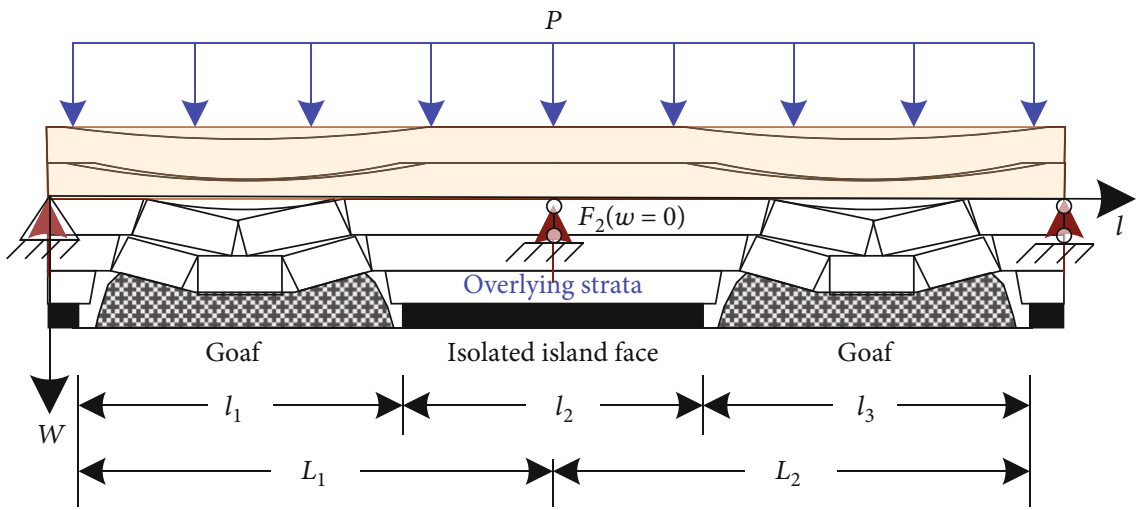

FIGURE 6: Continuous beam structure model of the overlying strata in the isolated island face.

shown in Figure 6. The bearing structure of overlying strata in the isolated island face and the gob on both sides is regarded as the continuous beam structure. The overlying stratum load is regarded as the uniformly distributed load on the upper part of the continuous beam structure. The coal-rock mass in the isolated island face and the coal-rock mass on the outer side of the gob on both sides are regarded as the three supporting points of the continuous beam structure to bear the overlying stratum load jointly. On the basis of the equivalent transfer of force, the total bearing load of coal-rock mass in the isolated island face is assumed to be equivalent to the hinged bearing point force in the middle of the continuous beam structure of the overlying strata. In accordance with this structural model, this study uses the theory of elastic-plastic mechanics to analyze the distribution of lateral mining stress in the isolated island face. The mechanical equations of the distribution of mining stress and the mining failure depth of the coal-rock mass in the isolated island face in fully mechanized top-coal caving mining are deduced.
The mechanical model is simplified to facilitate its solution and meet the engineering applicability. The following basic assumptions are made.

(1) The coal-rock mass in the isolated island face in fully mechanized top-coal caving mining is an ideal elastic-plastic medium, and the medium failure meets the Mohr-Coulomb strength criterion

(2) Before the formation of goafs on both sides of the mining area, all coal-rock masses are in the original rock stress environment

(3) The peak value of mining stress concentration is located at the boundary of the elastic and failure areas of coal-rock media

(4) The unstable bearing effect of goafs is disregarded

(5) On the basis of the bearing structure model of the continuous beam in the overlying strata of the 
isolated island face in fully mechanized top-coal caving mining, the internal supporting point of the continuous beam model is considered to be located in the middle of the coal-rock mass in the isolated island face. The lateral mining stress carried by the coalrock mass in the isolated island face is symmetrically distributed in the middle supporting point

(6) The end supporting point of the continuous beam model is considered to be located at the lateral mining failure boundary of the coal-rock mass on both sides of goafs

4.2. Analysis of Lateral Mining Stress in the Coal-Rock Mass in the Isolated Island Face. In accordance with the abovementioned basic assumptions, as shown in Figure 6, mechanical analysis of the bearing structure model of the continuous beam in the overlying strata of the isolated island face is performed. The bearing structure of the continuous beam of the overlying strata in the isolated island face is regarded as the research object. The continuous beam structure is a two-span statically indeterminate structure with an overall span of $L$, and the three fulcrums are located outside the goafs on both sides and in the middle of the isolated island face. The distance between the middle fulcrum and the two fulcrums is $L_{1}$ and $L_{2}$.

The statically indeterminate problem of the continuous beam is solved in accordance with structural mechanics [28]. The mechanical analysis of the continuous beam structure of the overlying strata in the isolated island face is conducted on the basis of the deformation coordination condition and superposition principle. The coordinate system of the beam structure model regards the left fulcrum as the origin, with the right horizontal direction $l$-axis and the downward vertical direction $w$-axis. Under the action of uniformly distributed load $P$ on the upper boundary, $w_{P}$ equation of the winding degree of a beam member is as follows:

$$
w_{P}=\frac{P}{24 E J}\left(2 L x^{2}-x^{3}-L^{3}\right) x
$$

where $P$ is the uniform load in the continuous beam structure, regarded as the original rock stress of the coal seam, $\mathrm{MPa} E$ is the elastic modulus of the beam structure, GPa; $J$ is the moment of inertia of the cross section of the beam structure, $\mathrm{m}^{4} ; L=l_{1}+l_{2}+l_{3} ; l_{2}$ is the lateral length of the isolated island face (including coal pillars on both sides), $\mathrm{m}$; and $l_{1}$ and $l_{3}$ are the lateral lengths of goafs on both sides (including the failure depth of the outer coal body), $\mathrm{m}$.

In the middle of the isolated island face of the beam structure model, with $F_{2}$ provided by the outer and middle fulcrum positions, the deflection $w_{F}$ equation of the beam structure is as follows:

$$
w_{F}=\left\{\begin{array}{l}
-\frac{F_{2} L_{2}}{6 E J L}\left(x^{2}-L^{2}+L_{2}^{2}\right) x,\left(0 \leq x \leq L_{1}\right), \\
-\frac{F_{2} L_{1}}{6 E J L}\left(x^{2}-2 L x+L_{1}^{2}\right)(L-x), \quad\left(L_{1} \leq x \leq L\right),
\end{array}\right.
$$

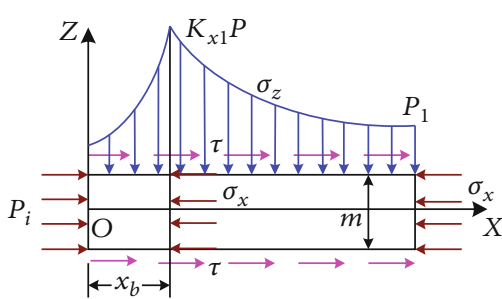

FIgURE 7: Stress analysis model of the edge coal-rock mass.

where $F_{2}$ is the concentrated reaction formed by the coalrock mass in the isolated island face, $N ; w_{F}$ is the bending degree of the concentrated reaction force $F_{2}$ to the beam structure, $\mathrm{m} ; L_{1}=l_{1}+l_{2} / 2 ; L_{2}=l_{2} / 2+l_{3}$.

The deformation coordination condition indicates that at the middle fulcrum position $\left(x=L_{1}\right),\left.w_{P}\right|_{x=L_{1}}+\left.w_{F}\right|_{x=L_{1}}=0$; it is combined with Equations (1) and (2). The solution is as follows:

$$
\frac{P}{24 E J}\left(2 L L_{1}^{2}-L_{1}^{3}-L^{3}\right) L_{1}=\frac{F_{2} L_{2}}{6 E J L}\left(L_{1}^{2}-L^{2}+L_{2}^{2}\right) L_{1} .
$$

The results are as follows:

$$
F_{2}=\frac{P L\left(L^{2}+L_{1}^{3}-2 L L_{1}^{2}\right)}{8 L_{2}^{2} L} .
$$

In view of the lateral force state of the coal-rock mass in the isolated island face in fully mechanized top-coal caving mining, the lateral mining results in the mining stress concentration at the edge of the lateral coal-rock mass. This phenomenon leads to the failure of coal-rock mass in the lateral edge and forms the failure area in the edge of the coal-rock mass in the isolated island face. From the literature [15-18] on the analysis of mining stress in the coal-rock mass in working face, elastic-plastic mechanics theory and the limit equilibrium method $[26,27$, 29] are used to analyze the mechanical failure of the coal-rock mass in the isolated island face, as shown in Figure 7.

In accordance with the basic assumption (5), the lateral mining stress carried by the coal-rock mass in the isolated island face is symmetrically distributed with the central supporting point. Therefore, microelement is considered for the coalrock mass in the isolated island face. Stress analysis is conducted with the microelement body as the research object. The lateral edge coal-rock mass boundary is regarded as the origin, and the $X$-axis in the deep horizontal direction and the $Z$-axis in the downward vertical direction are used to establish the mining dynamic analysis coordinate system of the lateral edge coalrock mass. The microelement body is mainly affected by the mining vertical stress $\sigma_{z}$ transferred from the overlying strata, the horizontal resistance $P_{i}$ on the lateral coal-rock boundary, normal stress $\sigma_{x}$, and the tangential stress $\tau$ produced by the upper and lower boundary dislocation. Thus, the force balance equation of the microelement body is established as follows:

$$
-m\left(\sigma_{x}+d \sigma_{x}\right)+m \sigma_{x}+2 \tau d x=0,
$$

where $m$ is the thickness of the coal-rock mass at the lateral edge, $\mathrm{m} ; \sigma_{x}$ is the horizontal stress in the lateral edge coal-rock 
mass, $\mathrm{MPa}$; and $\tau$ is the tangential stress formed by the upper and lower boundary dislocation, $\mathrm{MPa}$.

The tangential stress caused by the dislocation of the upper and lower boundaries of the lateral margin coal-rock mass is expressed as follows:

$$
\tau=f d \sigma_{z}
$$

where $\sigma_{z}$ is the vertical stress on the lateral edge coal-rock mass, $\mathrm{MPa}$; and $f$ is the friction resistance coefficient of lateral coal-rock boundary dislocation.

Equations (5) and (6) are combined to obtain

$$
d \sigma_{x}=\frac{2 f}{m} \sigma_{z} d x
$$

Let $\beta=2 \mathrm{f} / \mathrm{m}$ and the lateral coal-rock mass media meet the limit equilibrium conditions, as shown as follows:

$$
\sigma_{z}^{b}=K_{p} \sigma_{x}^{b}+\sigma_{c}
$$

where $\sigma_{x}^{b}$ and $\sigma_{z}^{b}$ are the horizontal stress and vertical stress in the failure zone of lateral coal-rock mass, respectively, $\mathrm{MPa}$; $\sigma_{c}$ is the uniaxial compressive strength, $\mathrm{MPa} ; \sigma_{c}=2 C \cos \varphi /$ $(1-\sin \varphi) ; C$ is the cohesion, $\mathrm{MPa} ; P_{z}$ is the internal friction angle, ${ }^{\circ}$; and $K_{p}=(1+\sin \varphi) /(1-\sin \varphi)$.

Equation (8) is substituted into Equation (7), and the general solution of the differential equation is obtained as follows:

$$
\sigma_{x}^{b}=C_{1} e^{\beta K_{p} x}-\frac{\sigma_{c}}{K_{p}}
$$

In the position $(x=0)$, when the existence of horizontal resistance $P_{i}$ of the coal-rock mass at the lateral edge, namely, $\left.\quad \sigma_{x}^{b}\right|_{x=0}=P_{i}$, is considered and Equation (9) is substituted, the solution is as follows:

$$
C_{1}=P_{i}+\frac{\sigma_{c}}{K_{p}}
$$

where $P_{i}$ is the horizontal resistance of the coal rock mass boundary at the lateral edge, $\mathrm{MPa}$.

Therefore, Equations (8) and (9) are used to solve the distribution equation of the lateral mining stress in the lateral failure area of coal-rock mass, as shown as follows:

$$
\left\{\begin{array}{l}
\sigma_{x}^{b}=\left(P_{i}+\frac{\sigma_{c}}{K_{p}}\right) e^{\beta K_{p} x}-\frac{\sigma_{c}}{K_{p}}, \\
\sigma_{z}^{b}=K_{p}\left(P_{i}+\frac{\sigma_{c}}{K_{p}}\right) e^{\beta K_{p} x},
\end{array}\right.
$$

where $x_{b}$ is the boundary between the lateral failure zone of coal-rock mass and the elastic zone, i.e., the failure depth of lateral mining, $\mathrm{m}$.
On the basis of the literature $[15-18,26,27,29]$ on the analysis of the convergence characteristics of mining stress in the elastic area of coal-rock mass, the distribution equation of lateral mining stress in the elastic area of the isolated island face is set as follows:

$$
\left\{\begin{array}{l}
\sigma_{x}^{e}=P_{1}-\frac{Q_{1}}{x^{2}}, \\
\sigma_{z}^{e}=P_{1}+\frac{Q_{1}}{x^{2}}
\end{array} \quad\left(x_{b} \leq x \leq \frac{l_{2}}{2}\right)\right.
$$

where $\sigma_{x}^{e}$ and $\sigma_{z}^{e}$ are the horizontal and vertical mining stresses, respectively, in the lateral elastic area of coal-rock mass, $\mathrm{MPa}$; and $P_{1}$ and $Q_{1}$ are the undetermined coefficients.

In the position $\left(x=x_{b}\right)$, the mining vertical and horizontal stresses in the elastic zone of the lateral edge coal-rock mass meet the limit equilibrium conditions, namely, $\sigma_{z}^{e}$ $\left.\right|_{x=x_{b}}=\left.K_{P} \sigma_{x}^{e}\right|_{x=x_{b}}+\sigma_{c}$.

From the stress transfer of continuous medium, we can determine $\left.\sigma_{z}^{e}\right|_{x=x_{b}}=\left.\sigma_{z}^{b}\right|_{x=x_{b}} ;\left.\sigma_{x}^{e}\right|_{x=x_{b}}=\left.\sigma_{x}^{b}\right|_{x=x_{b}}$.

The three boundary conditions in Equations (11) and (12) are combined to obtain

$$
\left\{\begin{array}{l}
P_{1}=\frac{\left(K_{p}+1\right)}{2}\left(P_{i}+\frac{\sigma_{c}}{K_{p}}\right) e^{\beta K_{p} x_{b}}-\frac{\sigma_{c}}{2 K_{p}}, \\
Q_{1}=\left[K_{p}\left(P_{i}+\frac{\sigma_{c}}{K_{p}}\right) e^{\beta K_{p} x_{b}}-P_{1}\right] x_{b}^{2} .
\end{array}\right.
$$

Therefore, the distribution equation of the lateral mining stress in the elastic area of the isolated island face is obtained as follows:

$$
\left\{\begin{array}{l}
\sigma_{x}^{e}=\left[\frac{\left(K_{p}+1\right)}{2}\left(P_{i}+\frac{\sigma_{c}}{K_{p}}\right) e^{\beta K_{p} x_{b}}-\frac{\sigma_{c}}{2 K_{p}}\right]\left(1+\frac{x_{b}^{2}}{x^{2}}\right)-K_{p}\left(P_{i}+\frac{\sigma_{c}}{K_{p}}\right) e^{\beta K_{p} x_{b}} \frac{x_{b}^{2}}{x^{2}}, \\
\sigma_{z}^{e}=\left[\frac{\left(K_{p}+1\right)}{2}\left(P_{i}+\frac{\sigma_{c}}{K_{p}}\right) e^{\beta K_{p} x_{b}}-\frac{\sigma_{c}}{2 K_{p}}\right]\left(1-\frac{x_{b}^{2}}{x^{2}}\right)+K_{p}\left(P_{i}+\frac{\sigma_{c}}{K_{p}}\right) e^{\beta K_{p} x_{b}} \frac{x_{b}^{2}}{x^{2}},
\end{array}\right.
$$

On the basis of the bearing structure model of the continuous beam of the overlying strata in the isolated island face in fully mechanized top-coal caving mining and the basic assumption (5), the lateral mining force carried by the coal-rock mass in the isolated island face is symmetrically distributed at the supporting point of the middle isolated island face. The load transfer balance equation of the lateral mining superposition influence is established by integrating

$$
\int_{x_{b}}^{l_{2} / 2} \sigma_{z}^{e} d x+\int_{0}^{x_{b}} \sigma_{z}^{b} d x=\frac{F_{2}}{2} .
$$




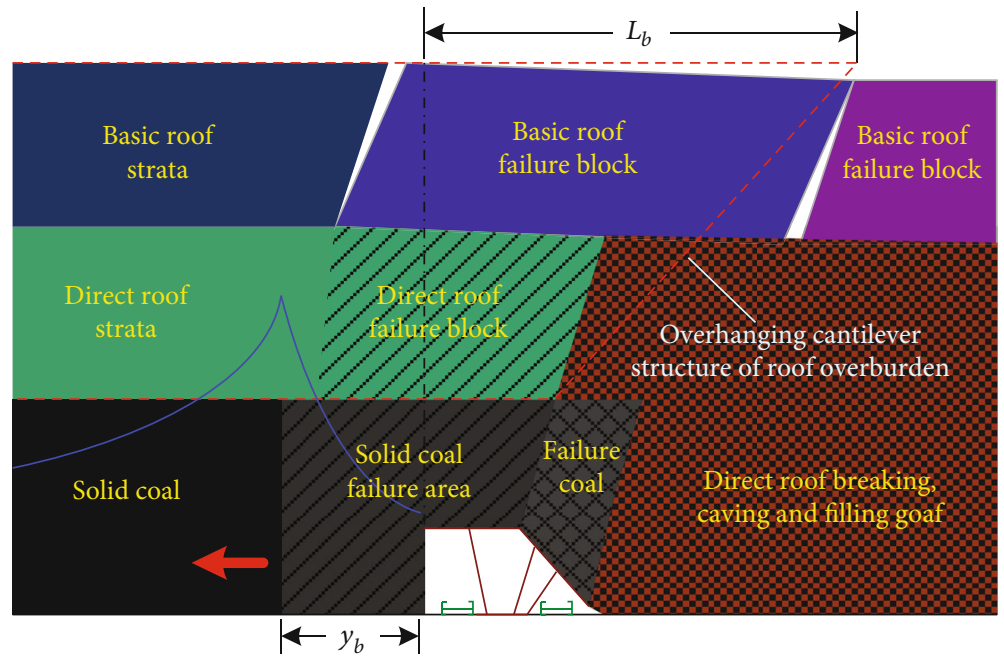

Figure 8: Cantilever structure characteristics of the coal wall roof in the isolated island face in fully mechanized top-coal caving mining.

Equations (4), (11), and (14) are substituted into Equation (15), and we have

$$
\begin{aligned}
& {\left[\frac{\left(K_{p}+1\right)}{2}\left(P_{i}+\frac{\sigma_{c}}{K_{p}}\right) e^{\beta K_{p} x_{b}}-\frac{\sigma_{c}}{2 K_{p}}\right] \frac{\left(l_{2}-2 x_{b}\right)^{2}}{2 l_{2}}} \\
& \quad+K_{p}\left(P_{i}+\frac{\sigma_{c}}{K_{p}}\right) e^{\beta K_{p} x_{b}} \frac{\left(l_{2}-2 x_{b}\right) x_{b}}{l_{2}} \\
& \quad+\frac{1}{\beta}\left(P_{i}+\frac{\sigma_{c}}{K_{p}}\right)\left(e^{\beta K_{p} x_{b}}-1\right)=\frac{P L\left(L^{2}+L_{1}^{3}-2 L L_{1}^{2}\right)}{16 L_{2}^{2} L} .
\end{aligned}
$$

The only unknown solution of the equation is the lateral mining failure depth $x_{b}$ of the isolated island face. The parameter can be calculated via numerical interpolation of Equation (16). $x_{b}$ can be substituted into Equation (14) to obtain the lateral mining stress distribution equation of the failure and elastic areas of the isolated island face. Equation (16) indicates that the lateral arrangement of the isolated island face (i.e., the lateral length $l_{2}$ of the isolated island face and the lateral lengths $l_{1}$ and $l_{3}$ of the goaf on both sides) has a remarkable effect on its lateral mining failure depth $x_{b}$ and influences the lateral mining superimposed stress distribution.

4.3. Mechanical Analysis of Advance Mining Stress in Front of the Coal Wall of the Isolated Island Face. Under the lateral mining superposition influence, mechanical analysis of mining stress state in front of coal wall in the mining process of the isolated island face is performed. The stress analysis of advance mining is consistent with the dynamic analysis principle of lateral mining. The stress analysis of advance mining is based on the back mining boundary of isolated island face as the origin, the direction of coal wall mining is $Y$-axis, and the vertical direction $Z$-axis is upward. Then, the analytical coordinate system of advance mining power of coal-rock mass in front of coal wall is established. Along the mining direction ( $Y$ direction) of the isolated island face, microelement is considered for the coal-rock mass in front of the back mining wall of the isolated island face. In the failure area of the edge of coal wall, the media of coal-rock mass meet the limit equilibrium condition in Equation (8). The stress distribution equation of the advance mining failure area is obtained as follows:

$$
\sigma_{z}^{e}=P_{z}(x)+\frac{Q_{2}}{y^{2}}, \quad\left(y_{b} \leq y\right)
$$

where $\sigma_{y}^{b}$ and $\sigma_{z}^{b}$ are the horizontal stress and vertical stress of the coal wall failure zone under the influence of advance mining, respectively, $\mathrm{MPa} ; P_{j}$ is the horizontal resistance of the mining coal wall in the isolated island face, MPa; and $y_{b}$ is the boundary between the failure and elastic areas in front of the mining coal wall, which is the advance mining failure depth of the coal wall.

In the elastic area of the coal-rock mass in front of the coal wall of the isolated island face, the influence of mining far away from the coal wall along the mining direction gradually converges to the lateral mining stress state. That is, the vertical stress distribution of the coal-rock mass in front of the coal wall of the isolated island face is $\left.\sigma_{z}^{e}\right|_{y=+\infty}=P_{z}(x)$, where $P_{z}(x)$ is the vertical stress at $x$ position before the influence of advance mining, i.e., $P_{z}(x)=\sigma_{z}^{e}(x)=\left[\left(\left(K_{p}+1\right) / 2\right)(\right.$ $\left.\left.P_{i}+\left(\sigma_{c} / K_{p}\right)\right) e^{\beta K_{p} x_{b}}-\left(\sigma_{c} / 2 K_{p}\right)\right]\left(1-\left(x_{b}^{2} / x^{2}\right)\right)+K_{p}\left(P_{i}+\left(\sigma_{c} l\right.\right.$ $\left.\left.K_{p}\right)\right) e^{\beta K_{p} x_{b}}\left(x_{b}^{2} / x^{2}\right)$.

Then, the mining vertical stress equation of the elastic area in front of the coal wall of the isolated island face is set as follows:

$$
\sigma_{z}^{e}=P_{z}(x)+\frac{Q_{2}}{y^{2}}, \quad\left(y_{b} \leq y\right),
$$

where $Q_{2}$ is the coefficient to be determined. 


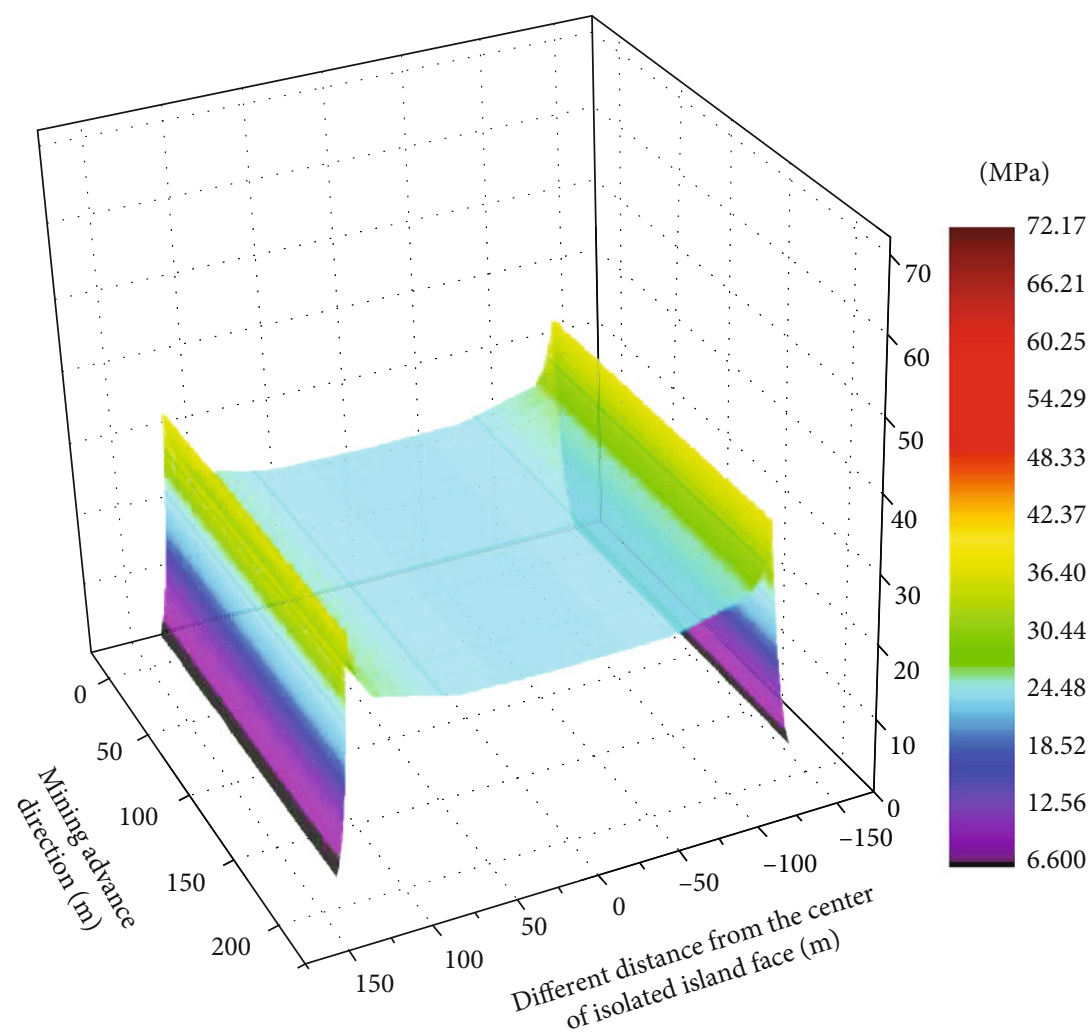

FIGURE 9: Characteristics of the distribution of lateral mining stress in the isolated coal face.

At the advance mining failure boundary $\left(y=y_{b}\right)$ of the coal wall of the isolated island face, from the stress transfer of continuous medium, we can determine that $\left.\sigma_{z}^{e}\right|_{y=y_{b}}=\left.\sigma_{z}^{b}\right|_{y=y_{b}}$.

If the boundary conditions in Equations (17) and (18) are combined, the solution is as follows:

$$
Q_{2}=\left[K_{p}\left(P_{j}+\frac{\sigma_{c}}{K_{p}}\right) e^{\beta K_{p} y_{b}}-P_{z}(x)\right] y_{b}^{2} .
$$

In accordance with masonry beam theory and rock beam transmission theory in the literature $[28,29]$, this paper reveals the characteristics and periodic pressure of the fracture structure in the overlying strata in the isolated island face. During the mining process of the isolated island face, the roof overlying strata of the coal wall present the characteristics of cantilever structure and periodic breaking pressure, as shown in Figure 8.

The cantilever structure length of coal wall roof affects the advance mining stress distribution in front of the coal wall and leads to the appearance of periodic pressure characteristics. Therefore, in the mechanical model analysis, the cantilever structure length is regarded as the periodic pressing step distance $L_{b}$. On the basis of the principle of force equivalence, the balance equation of force transfer and bearing is established via integration, as shown as follows:

$$
\int_{y_{b}}^{+\infty} \sigma_{z}^{e} d y+\int_{0}^{y_{b}} \sigma_{z}^{b} d y=\int_{-L_{b}}^{+\infty} P_{z}(x) d y
$$

where $L_{b}$ is the length of the overhanging cantilever structure of roof overburden, $\mathrm{m}$.

Equations (17) and (18) are substituted into Equation (20), and the solution is as follows:

$$
K_{p} y_{b}\left(P_{j}+\frac{\sigma_{c}}{K_{p}}\right) e^{\beta K_{p} y_{b}}-2 P_{z}(x) y_{b}+\frac{1}{\beta}\left(P_{j}+\frac{\sigma_{c}}{K_{p}}\right)\left(e^{\beta K_{p} y_{b}}-1\right)=P_{z}(x) L_{b} .
$$

Accordingly, the advance mining failure depth $y_{b}$ can be obtained via numerical interpolation. The vertical stress equation of mining in the elastic area in the isolated island face can be determined by substituting $y_{b}$ into Equation (18), i.e.,

$$
\begin{aligned}
\sigma_{z}^{e}= & {\left[\frac{\left(K_{p}+1\right)}{2}\left(P_{i}+\frac{\sigma_{c}}{K_{p}}\right) e^{\beta K_{p} x_{b}}-\frac{\sigma_{c}}{2 K_{p}}\right]\left(1-\frac{x_{b}^{2}}{x^{2}}\right)\left(1-\frac{y_{b}^{2}}{y^{2}}\right) } \\
& +K_{p}\left(P_{i}+\frac{\sigma_{c}}{K_{p}}\right) e^{\beta K_{p} x_{b}} \frac{x_{b}^{2}}{x^{2}}\left(1-\frac{y_{b}^{2}}{y^{2}}\right) \\
& +K_{p}\left(P_{j}+\frac{\sigma_{c}}{K_{p}}\right) e^{\beta K_{p} y_{b}} \frac{y_{b}^{2}}{y^{2}}, \quad\left(y_{b} \leq y\right) .
\end{aligned}
$$

The advance mining depth in front of the coal wall increases because of the advance mining influence of the 


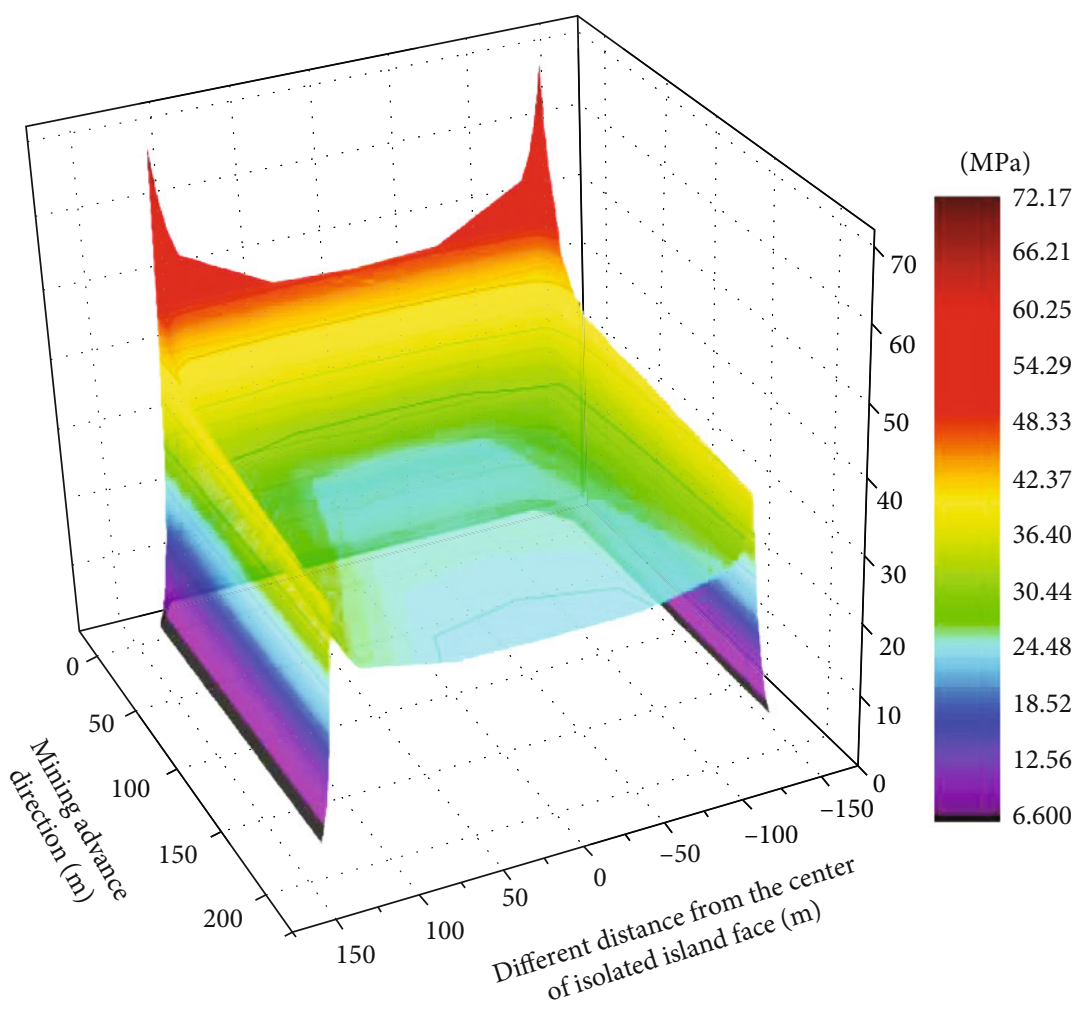

FIGURE 10: Characteristics of the distribution of advance mining stress in the isolated coal face.

isolated island face. Consequently, the advance mining stress distribution changes.

Therefore, the advance mining stress and failure of coalrock mass should be recalculated in combination with the mining influence in front of the mining coal wall of the isolated island face. When $\left.\sigma_{z}^{e}\right|_{x=x_{b}}$ from Equation (22) is substituted into Equation (11), the equation of lateral mining failure depth under the influence of advance mining of the isolated island face is obtained as follows:

$$
x_{B}=\frac{1}{\beta K_{p}} \ln \left[\frac{\left(K_{p} P_{i}+\sigma_{c}\right)}{\left(K_{p} P_{j}+\sigma_{c}\right)} e^{\beta K_{p} x_{b}}\left(1-\frac{y_{b}^{2}}{y^{2}}\right)+e^{\beta K_{p} y_{b}} \frac{y_{b}^{2}}{y^{2}}\right] .
$$

$x_{B}$ obtained from Equation (23) is substituted into Equations (11) and (14) as new $x_{b}$ for recalculation. The distribution of mining stress in the isolated island face is obtained.

\section{Engineering Application Analysis}

5.1. Example Analysis of the Distribution of Mining Stress in the 61102 Isolated Island Face in Fully Mechanized TopCoal Caving Mining. The 61102 isolated island face in fully mechanized top-coal caving mining is regarded as the engineering research object. The initial conditions of the example analysis are as follows: $P=14 \mathrm{MPa}, m=18.3 \mathrm{~m}, C=1.3 \mathrm{MPa}$, $\varphi=28^{\circ}, f=0.53, P_{i}=0 \mathrm{MPa}$, and $P_{j}=0 \mathrm{MPa}$. The lateral length of the 61102 isolated island face (including the coal pillars at both sides) is $l_{2}=280 \mathrm{~m}$; the lateral lengths of 61103 and
61101 working face (including the lateral failure depth of $15 \mathrm{~m}$ ) are $l_{1}=230 \mathrm{~m}$ and $l_{3}=250 \mathrm{~m}$, respectively; the cantilever structure of coal wall and roof is $L_{b}=12.0 \mathrm{~m}$ (the approximate pressure step distance of the 61102 isolated island face in the peripheral period). The aforementioned parameters are integrated into the mechanical equation of mining stress in the isolated island face. $x_{b}$ solved by Equation (16) is substituted into Equations (11) and (14). The coordinate is converted symmetrically to obtain the distribution of mining stress in the isolated island face. The distribution of lateral mining stress in the isolated island face is shown in Figure 9. $y_{b}$ is obtained from Equation (21), which is substituted into Equations (17), (22), and (23). $x_{B}$ in Equations (11) and (14) is replaced with $x_{b}$ obtained from Equation (23). The distribution of lateral mining stress in the isolated island face is recalculated. The distribution of mining stress in front of the mining wall of the isolated island face is shown in Figure 10.

As shown in Figure 9, the 61102 isolated island face in fully mechanized top-coal caving mining is affected by the lateral mining superposition influence of bilateral goafs. The mining stress concentration is formed at the side edge of the coal-rock mass in the 61102 isolated island face. The peak value of the mining stress is $36 \mathrm{MPa}$, the mining stress concentration coefficient is nearly 2.6, the lateral mining failure depth is $13.2 \mathrm{~m}$, and the depth bearing mining stress is generally approximately 23.5 $\mathrm{MPa}$ (up to 1.67 times of the original rock stress).

In Figure 10, in the mining process of the 61102 isolated island face, from the middle to the both ends of the mining coal wall, the advance mining stress concentration is $48.2-$ 72.2 $\mathrm{MPa}$, the advance mining stress concentration 


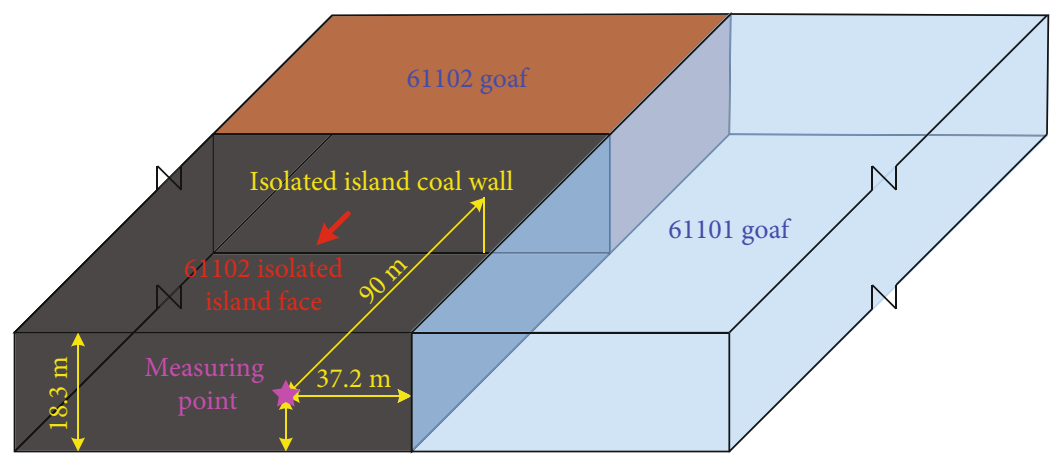

FIGURE 11: Measuring point location for advance mining stress in the isolated island face.

coefficient is 3.3-5.0, and the advance mining failure depth is $15.0-17.5 \mathrm{~m}$. In the upper and lower ends, the lateral mining failure depth is up to $17.5 \mathrm{~m}$, and the influence of mining is most severe in the range of $30 \mathrm{~m}$ from top to bottom.

Theoretical calculation and analysis show that the stress concentration coefficient of lateral mining superposition influence of the isolated island face is 2.6, and the lateral deep bearing stress is 1.67 times of the original rock stress. During the mining process of the isolated island face, the advance mining stress concentration coefficient of the upper and lower ends is as high as 5.0. The lateral mining failure depth increases by $2.0-5.0 \mathrm{~m}$ under the advance mining influence in the isolated island face. The mining influence in the range of 20-30 $\mathrm{m}$ of the upper and lower ends is intense, and the mining stress in this area is characterized by "cone distribution." Therefore, this area is the key bearing structure of mining stress concentration in the isolated island face. The advance mining stress concentration of the isolated island face should be determined, and the support strength of coal-rock and the working resistance of the isolated island face support should be appropriately improved to avoid the instability of the bearing structure of coal-rock.

5.2. Comparison and Evaluation of Measured Results. The borehole stress meter is arranged in the coal body of the 61102 isolated island face. The layout of measuring points is shown in Figure 11. The measured results are compared with the theoretical calculation and numerical simulation results, as presented in Figure 12.

The measured results show that the peak value of the advance mining stress in the coal body of the 61102 isolated island is nearly $36 \mathrm{MPa}$ and $14 \mathrm{~m}$ away from the coal wall. The supporting load at $25 \mathrm{~m}$ away from the mining coal wall is nearly $20 \mathrm{MPa}$, and the stress distribution tends to be stable. The theoretical calculation results show that the peak value of the advance mining stress is $53.6 \mathrm{MPa}$, and it is close to $17.5 \mathrm{~m}$ from the mining coal wall. The supporting load outside at $40 \mathrm{~m}$ tends to stabilize at $30 \mathrm{MPa}$. The numerical simulation shows that the peak value of the advance mining stress is $52.6 \mathrm{MPa}$, and it is within $15-17 \mathrm{~m}$ from the mining coal wall. The supporting load outside at $40 \mathrm{~m}$ tends to stabilize at $35 \mathrm{MPa}$. Comparative analysis and verification imply that the theoretical calculation is basically consistent with the distribution trend of advance mining stress in the numerical simulation and field measurement. The mechanical

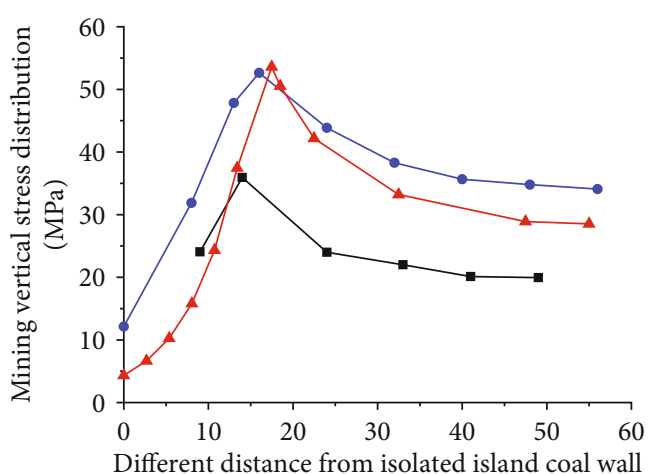

$(\mathrm{m})$

- Field measured curve

$\rightarrow$ Numerical simulation curve

_- Theoretical calculation curve

Figure 12: Contrast of the distribution of advance abutment pressure in the 61102 isolated island face in fully mechanized topcoal caving mining.

equation of the mining stress distribution and failure depth of the isolated island face is derived. It provides a timely and effective calculation method for the estimation of mining stress distribution and failure depth of the isolated island face and plays a certain engineering guiding role in the safe production decision making of the isolated island face in fully mechanized top-coal caving mining.

5.3. Analysis on Mining Stress Influence Factors in the Isolated Island Face of Extra-Thick Fully Mechanized Top-Coal Caving Mining. Taking the 61102 isolated island face condition in Tangjiahui Coal Mine as the engineering background, the influencing factors and laws of mining stress transfer on isolated island face are analyzed.

For mining conditions, as shown in Figure 13(a), the greater the mining height is, the smaller the mining stress concentration before and after the influence of advanced mining on isolated island face is, but it increases the lateral load of coal body, leading to the more serious mining failure of isolated island face, and as shown in Figures 13(b)-13(d), the smaller the width of isolated island face is, the more serious the influence degree of lateral mining superimposition will be, and the larger the difference of goaf width on both sides is, the more serious the mining load on isolated island face is; all of these lead to more serious mining stress 


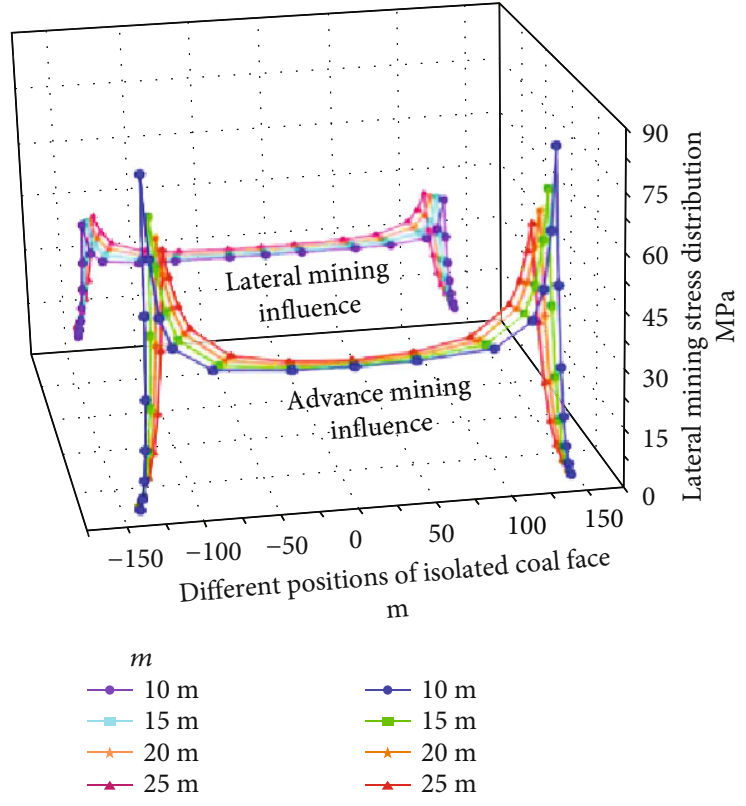

(a) Different mining heights of coal seams $(m)$

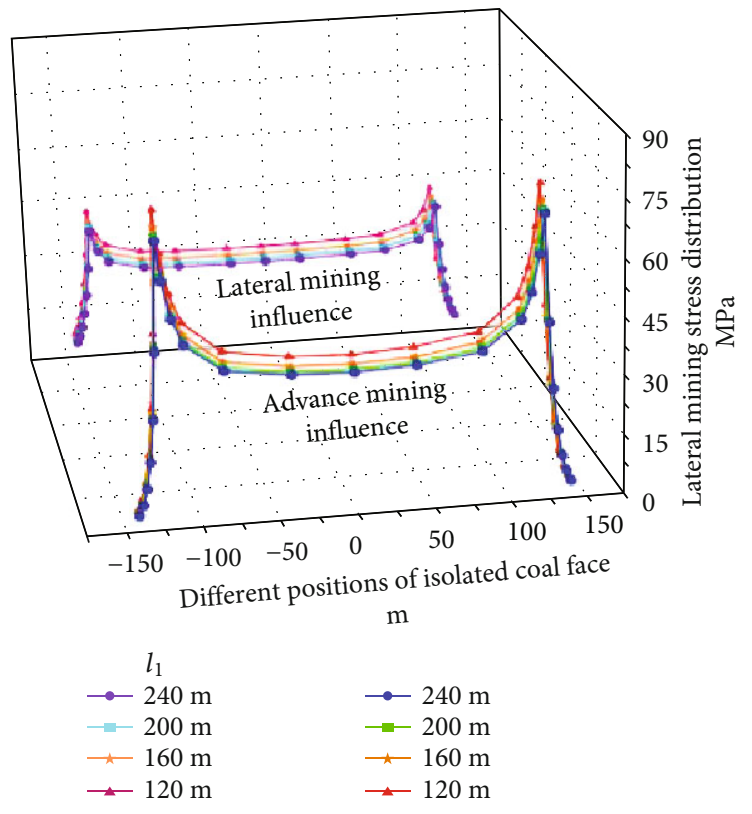

(c) Different goaf widths ( $l_{1}$ decreases)

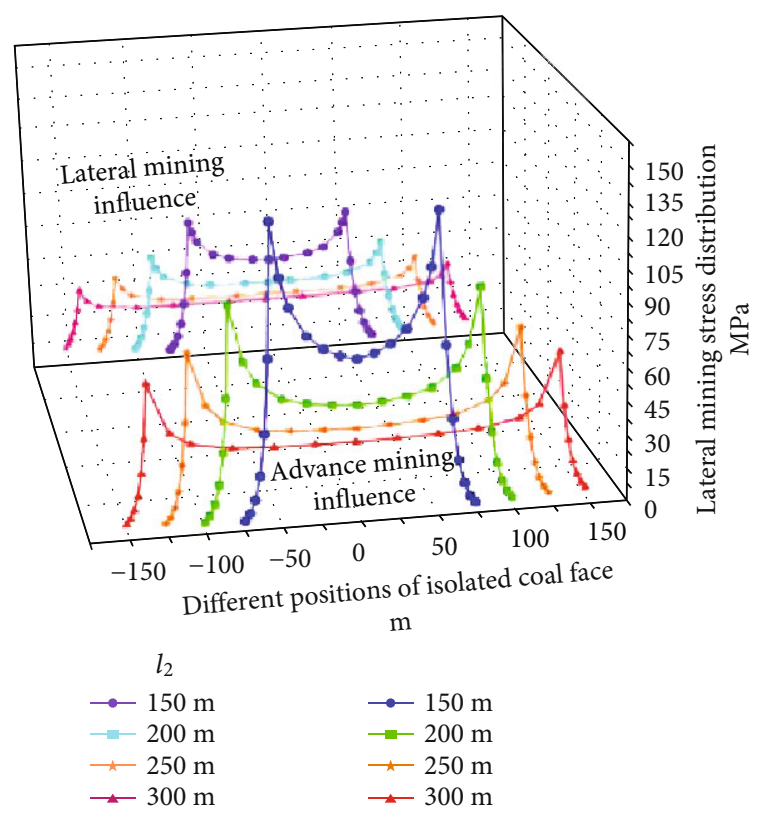

(b) Different isolated island widths $\left(l_{2}\right)$

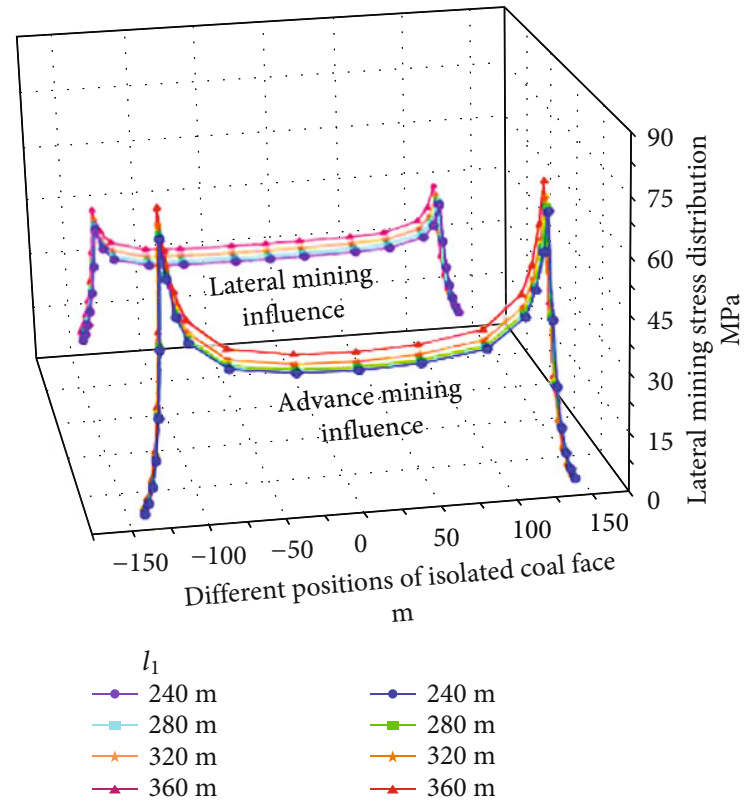

(d) Different goaf widths $\left(l_{1}\right.$ increases $)$

Figure 13: Continued. 


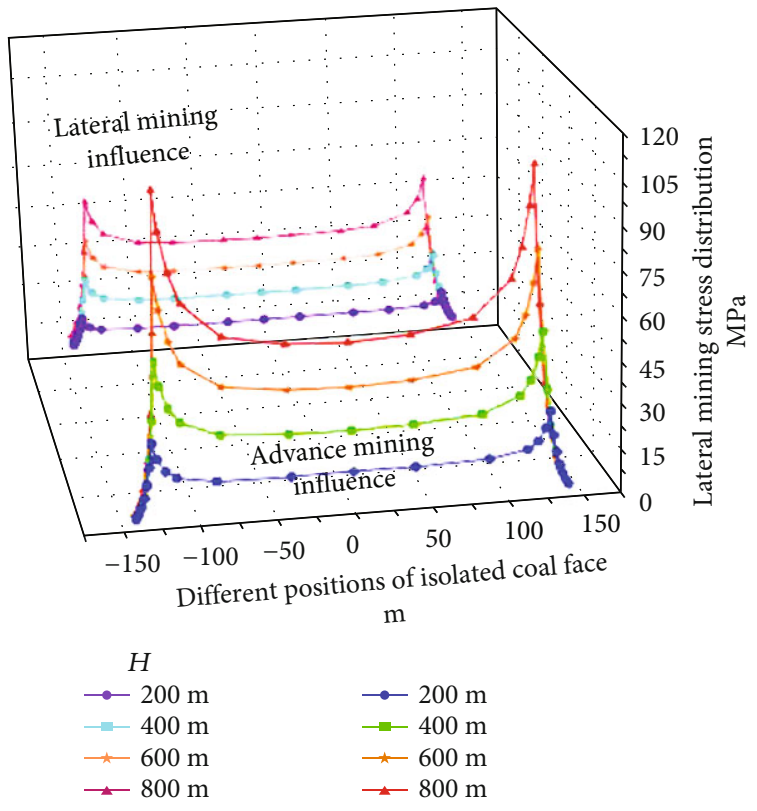

(e) Different depths of coal seam $(H)$

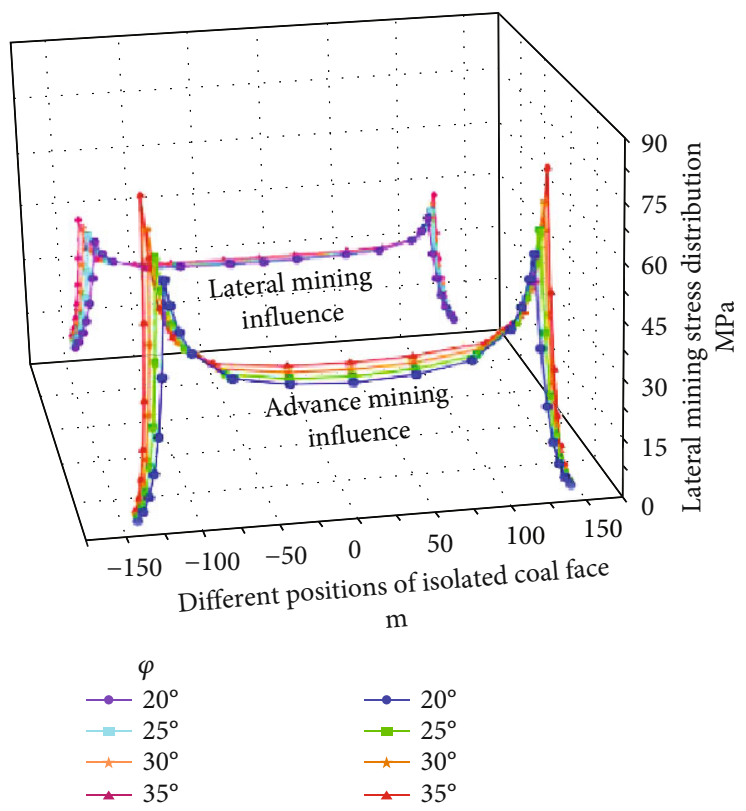

(g) Different internal friction angles of coal seam medium $(\varphi)$

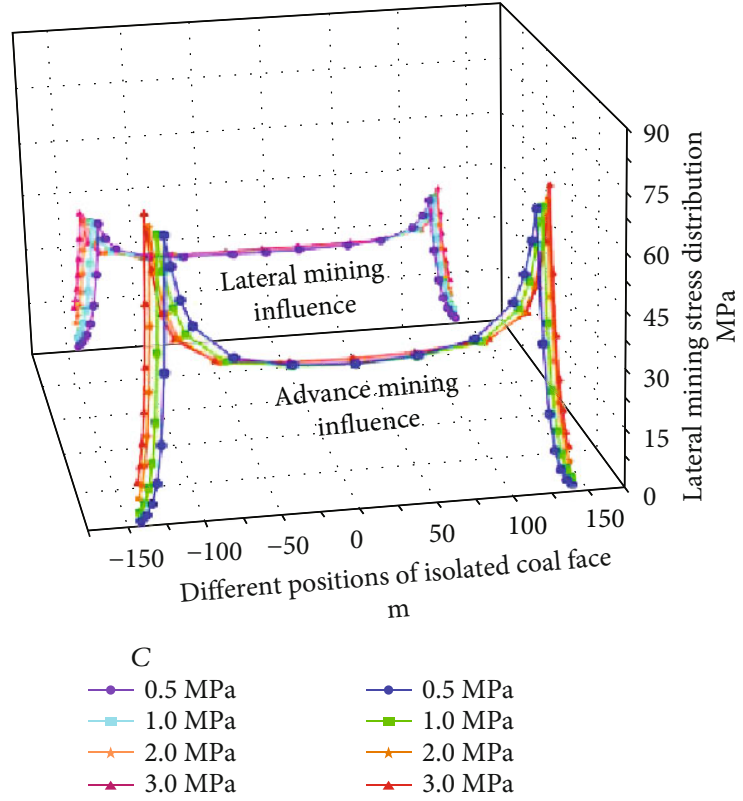

(f) Different cohesions of coal seam media $(C)$

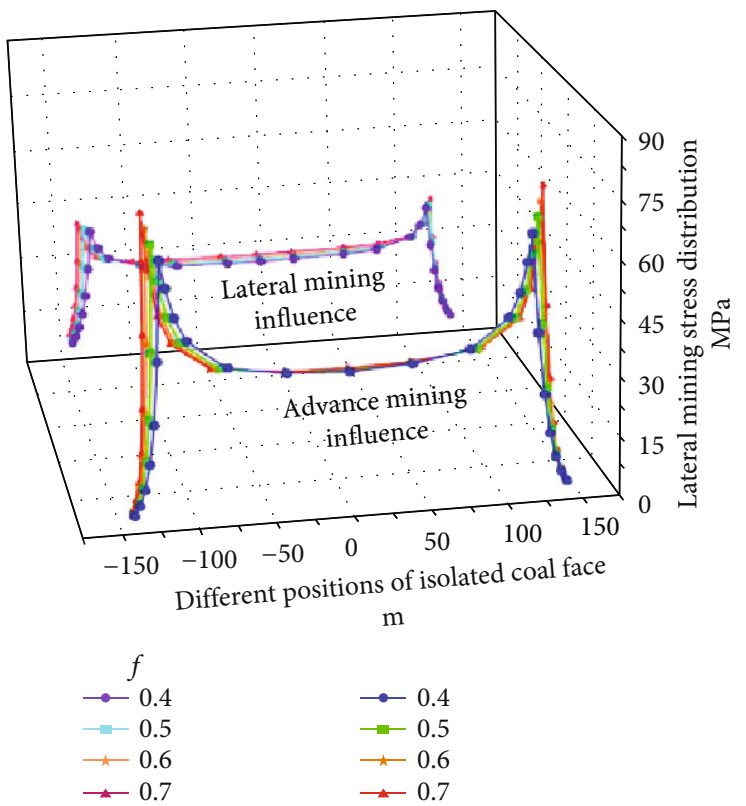

(h) Different friction coefficients on coal-rock upper and lower boundary $(f)$

FIGURE 13: Influence characteristics of various factors on mining stress of isolated island face.

concentration and failure degree of isolated island face. In terms of coal occurrence conditions, as shown in Figure 13(e), the greater the depth of coal seam is, the more serious the mining pressure appearance on isolated island face is; as shown in Figures 13(f)-13(h), the better the mechanical conditions of coal seam medium (i.e., the greater the cohesion, internal friction angle, and the staggered friction coefficient of upper and lower boundaries) are, the faster the convergence of mining stress is and the smaller the degree of mining failure, but it will form a higher mining stress concentration, leading to the greater hidden danger of induced scour disaster. Comprehensive analysis shows that the longer the isolated island face size is, the closer the goaf size on both sides of the isolated island face is, the smaller the coal seam buried depth is, the better the mechanical conditions of coal and rock medium are, and the smaller the mining height of coal seam is, the more favorable the safe mining of isolated island face is.

\section{Conclusion}

(1) The coal-rock mass in an isolated island face in fully mechanized top-coal caving mining is forced to be the "bearing and stress-transferring" structure of the overlying strata of bilateral goafs. The coal-rock mass should bear not only the lateral mining superposition 
influence but also the advance mining influence in front of the coal wall; therefore, the isolated island face is in the complex environment of multiple mining stress superpositions

(2) Compared with the nonisolated island face, the mining pressure appearance is intense. In the mining process, the maximum advance mining stress concentration factor is 4.0-6.0 and is located at the upper and lower ends of the isolated island face. The lateral mining failure depth of the coal wall of the isolated island face increases by 2.0-5.0 $\mathrm{m}$ under the influence of advance mining

(3) A serious mining influence area is formed under the superimposition of the lateral and advance mining influences of the isolated island face. The mining influence in the range of $20-30 \mathrm{~m}$ of the upper and lower ends is intense, and the mining stress in this area is characterized by "cone distribution." This zone is an important hidden danger area with coalrock mass mining instability on isolated island face

(4) According to the mining influencing factors and laws of isolated island face, the longer the isolated island face size is, the closer the goaf size on both sides of the isolated island face is, the smaller the coal seam buried depth is, the better the mechanical conditions of coal and rock medium are, and the smaller the mining height of coal seam is, the more favorable the safe mining of isolated island face is

(5) The mechanical model of the bearing structure of an overburden continuous beam on an island face is established. The mechanical equation of mining stress distribution and coal-rock mass mining failure is obtained using elastic-plastic mechanics theory and limit equilibrium method. It provides an estimation method for the mining dynamic analysis of isolated island face in extra-thick fully mechanized top-coal caving mining

\section{Data Availability}

The known data in this paper come from practical engineering case data, which are reliable and available.

\section{Conflicts of Interest}

The authors declare that there is no conflict of interest regarding the publication of this article.

\section{Acknowledgments}

This study was supported by the NSFC Project (51574007, 51674007), the Inner Mongolia Natural Science Foundation Project (2019MS05055), and the Open Research Fund Project of Key Laboratory of Safety and High-Efficiency Coal Mining of Ministry of Education (JYBSYS2019208).

\section{References}

[1] F. X. Jiang, X. M. Zhang, S. H. Yang, L. Xun, Q. Ma, and H. Wang, "Discussion on overlying strata spatial structures of long wall in coal mine," Chinese Journal of Rock Mechanics and Engineering, vol. 25, no. 5, pp. 979-984, 2006.

[2] F. X. Jiang, "Viewpoint of spatial structures of overlying strata and its application in coal mine," Journal of Mining \& Safety Engineering, vol. 23, no. 1, pp. 30-33, 2006.

[3] H. Shi, C. W. Wang, and L. H. Kong, "Mechanism discussion on rock bursting caused by coal pillar of S-shaped overlying multistage spatial structure," Chinese Journal of Rock Mechanics and Engineering, vol. 31, no. 2, pp. 3508-3513, 2012.

[4] Y. H. Cheng, F. X. Jiang, X. M. Zhang, Z. Y. Mao, and Z. W. Ji, "C-shaped strata spatial structure and stress field in long wall face monitored by micro seismic monitoring," Chinese Journal of Rock Mechanics and Engineering, vol. 26, no. 1, pp. 102-107, 2007.

[5] W. Hou, F. X. Jiang, C. W. Wang, Z. Q. Feng, and D. Z. Wang, "Pressure control in sublevel long face surrounded by three sides mined areas of C-shaped strata spatial structure," Journal of China Coal Society, vol. 34, no. 3, pp. 310-314, 2009.

[6] L. M. Dou and H. He, "Study of OX-F-T spatial structure evolution of overlying strata in coal mines," Chinese Journal of Rock Mechanics and Engineering, vol. 31, no. 3, pp. 453-460, 2012.

[7] L. M. Dou, Z. L. Mu, Z. L. Li, A. Y. Cao, and S. Y. Gong, "Research progress of monitoring, forecasting, and prevention of rockburst in underground coal mining in China," International Journal of Coal Science and Technology, vol. 1, no. 3, pp. 278-288, 2014.

[8] A. Y. Cao, L. L. Zhu, F. C. Li, L. M. Dou, Y. L. Zhao, and Z. L. Zhang, "Characteristics of T-type overburden structure and tremor activity in isolated face mining under thick-hard strata," Journal of China Coal Society, vol. 39, no. 2, pp. 328335, 2014.

[9] H. Wang, Y. Jiang, Y. Zhao, J. Zhu, and S. Liu, "Numerical investigation of the dynamic mechanical state of a coal pillar during longwall mining panel extraction," Rock Mechanics and Rock Engineering, vol. 46, no. 5, pp. 1211-1221, 2013.

[10] Y. Jiang, H. Wang, S. Xue, Y. Zhao, J. Zhu, and X. Pang, "Assessment and mitigation of coal bump risk during extraction of an island longwall panel," International Journal of Coal Geology, vol. 95, no. 2, pp. 20-33, 2012.

[11] X. Li, B. Song, and P. Li, "Study on optimization of mining pillar in isolated island working face of thick coal seam under complex conditions," IOP Conference Series Earth and Environmental ence, vol. 21, no. 5, pp. 1-8, 2019.

[12] X. C. Liang, M. M. Zhu, D. C. Lu, and X. Liu, "Strata pressure behavior study on isolated island face in Huainan mine area," Advanced Materials Research, vol. 8, no. 3, pp. 367-369, 2013.

[13] G. D. Zhao and L. J. Kang, "Reviews of the feature of mine pressure appear on coal pillar of full mechanized caving coal face among gobs," Journal of Xi'an University of Science \& Technology, vol. 20, no. 1, pp. 39-42, 2000.

[14] W. Xu, E. Wang, R. Shen, D. Song, and J. Zhang, "Distribution pattern of front abutment pressure of fully-mechanized working face of soft coal isolated island," International Journal of Mining Science \& Technology, vol. 22, no. 2, pp. 279-284, 2012.

[15] D. C. Wang, H. T. Wang, S. C. Li, Q. Wang, and Y. Song, "Stress and deformation analysis of the side wall in a roadway driven along goaf incorporating the softening characteristics of 
coal strength mass," Journal of China University of Mining \& Technology, vol. 48, no. 2, pp. 295-304, 2019.

[16] S. Jayanthu, T. N. Singh, and D. P. Singh, "Stress distribution during extraction of pillars in a thick coal seam," Rock Mechanics and Rock Engineering, vol. 37, no. 3, pp. 171-192, 2004.

[17] S. C. Jia, J. C. Wan, and J. M. Zhu, "Calculation and application on elastic-plastic coal pillar width of the stope," Metal Mine, vol. 26, pp. 1116-1124, 2011.

[18] J. C. Chang, G. X. Xie, and X. H. Zhang, "Analysis of rib spalling mechanism of fully-mechanized top-coal caving face with great mining height in extra-thick coal seam," Yantu Lixue/Rock and Soil Mechanics, vol. 36, no. 3, pp. 803-808, 2015.

[19] X. Y. Zhang and M. Tu, "Deformation and failure characteristics of surrounding rock in gob-side entry during mining between the upper and lower gob island," Chinese Journal of Underground Space and Engineering, vol. 11, no. 4, pp. 10721079, 2015.

[20] S. X. Zhou and H. B. Li Hong, "Study on rib spalling cause and control technology of high cutting island coal mining face," Coal Science and Technology, vol. 45, no. 5, pp. 98-102, 2017.

[21] Z. Q. Wang, C. H. Xu, Y. J. Ren et al., "Study on roof failure mechanism of isolated island working face under abutment pressure," Journal of Safety Science and Technology, vol. 15, no. 6, pp. 105-112, 2019.

[22] M. G. Qian, P. W. Shi, and J. L. Xu, Mine Pressure and Strata Control, China University of Mining and Technology Press, Beijing, China, 2009.

[23] M. F. Cai, M. C. He, and D. Y. Liu, Rock Mechanics and Engineering, Science Press, Beijing, China, 2002.

[24] D. M. Pappas and C. Mark, Behavior of Simulated Longwall Gob Material, US Department of the Interior, Bureau of Mines: Report of Investigations, Xuzhou, China, 1993.

[25] M. D. G. Salamon, "Mechanism of caving in longwall coal mining," Proceedings of 31th US Rock Mechanics Symposium, pp. 161-168, Denver. Rotterdam, 1999.

[26] Z. Zhang, J. Bai, Y. Chen, and S. Yan, “An innovative approach for gob-side entry retaining in highly gassy fully-mechanized longwall top-coal caving," International Journal of Rock Mechanics and Mining Sciences, vol. 80, pp. 1-11, 2015.

[27] X. Y. Wu, Evolutionary Regularity of Plastic Zone and Stability Control in Repetitive Mining Roadway in Shendong Mining Area, China University of Mining and Technology, Beijing, China, 2018.

[28] C. M. Zhu and W. P. Zhang, Structural Mechanics, Higher Education Press, Beijing, China, 2016.

[29] Z. L. Xu, Elasticity, Higher Education Press, Beijing, China, 2016. 